\title{
Data Analytics and Artificial Intelligence in Predicting Length of Stay, Readmission, and Mortality: A Population-Based Study of Surgical Management of Colorectal Cancer
}

\section{Shamsul Masum}

University of Portsmouth

Adrian Hopgood ( $\square$ adrian.hopgood@port.ac.uk)

University of Portsmouth

\section{Samuel Stefan}

Portsmouth Hospitals University NHS Trust

\section{Karen Flashman}

Portsmouth Hospitals University NHS Trust Jim Khan

Portsmouth Hospitals University NHS Trust

\section{Research Article}

Keywords: Colorectal cancer, Colorectal Surgery, Prediction, Predictor Variables, Length of stay, Readmission, Mortality, Data Analytics, Artificial Intelligence, Machine Learning

Posted Date: November 17th, 2021

DOI: https://doi.org/10.21203/rs.3.rs-1082636/v1

License: (c) (i) This work is licensed under a Creative Commons Attribution 4.0 International License.

Read Full License

Version of Record: A version of this preprint was published at Discover Oncology on February 28th, 2022. See the published version at https://doi.org/10.1007/s12672-022-00472-7. 


\title{
Data Analytics and Artificial Intelligence in Predicting Length of Stay, Readmission, and Mortality: A Population-Based Study of Surgical Management of Colorectal Cancer
}

\author{
Shamsul Masum 10 , Adrian Hopgood*1 (0), Samuel Stefan² (0), \\ Karen Flashman' ${ }^{2}$, and Jim Khan ${ }^{2} 30$ \\ ${ }^{1}$ Faculty of Technology, University of Portsmouth, Portland \\ Building, Portland Street, Portsmouth, PO1 3AH, UK \\ ${ }^{2}$ Colorectal Department, Portsmouth Hospitals University NHS \\ Trust, Southwick Hill Road, Portsmouth, PO6 3LY, UK \\ ${ }^{3}$ Faculty of Science \& Health, University of Portsmouth, St \\ Michael's Building, White Swan Road, Portsmouth, PO1 2DT, UK
}

November 15, 2021

${ }^{*}$ Corresponding author: Adrian Hopgood - adrian.hopgood@port.ac.uk 


\title{
Data Analytics and Artificial Intelligence in Predicting Length of Stay, Readmission, and Mortality: A Population-Based Study of Surgical Management of Colorectal Cancer
}

\author{
Shamsul Masum ${ }^{1}$, Adrian Hopgood ${ }^{1 *}$, Samuel Stefan ${ }^{2}$, Karen Flashman ${ }^{2}$ and Jim \\ $\operatorname{Khan}^{2,3}$ \\ ${ }^{1}$ Faculty of Technology, University of Portsmouth, Portland Building, Portland Street, \\ Portsmouth, PO1 3AH, UK. \\ ${ }^{2}$ Colorectal Department, Portsmouth Hospitals University NHS Trust, Southwick Hill \\ Road, Portsmouth, PO6 3LY, UK. \\ ${ }^{3}$ Faculty of Science \& Health, University of Portsmouth, St Michael's Building, White \\ Swan Road, Portsmouth, PO1 2DT, UK.
}

*Corresponding author(s). E-mail(s): adrian.hopgood@port.ac.uk;

Contributing authors: shamsul.masum@port.ac.uk; samuel.stefan@porthosp.nhs.uk; karen.flashman@porthosp.nhs.uk; jim.khan@porthosp.nhs.uk;

\begin{abstract}
Data analytics and artificial intelligence (AI) have been used to predict patient outcomes after colorectal cancer surgery. A prospectively maintained colorectal cancer database was used, covering 4336 patients who underwent colorectal cancer surgery between 2003 and 2019. The 47 patient parameters included demographics, peri- and post-operative outcomes, surgical approaches, complications, and mortality. Data analytics were used to compare the importance of each variable and AI prediction models were built for length of stay (LOS), readmission, and mortality. Accuracies of at least $80 \%$ have been achieved. The significant predictors of LOS were age, ASA grade, operative time, presence or absence of a stoma, robotic or laparoscopic approach to surgery, and complications. The model with support vector regressor (SVR) algorithms predicted the LOS with an accuracy of $83 \%$ and mean absolute error (MAE) of 9.69 days. The significant predictors of readmission were age, laparoscopic procedure, stoma performed, preoperative nodal (N) stage, operation time, operation mode, previous surgery type, LOS, and the specific procedure. A BI-LSTM model predicted readmission with $87.5 \%$ accuracy, $84 \%$ sensitivity, and $90 \%$ specificity. The significant predictors of mortality were age, ASA grade, BMI, the formation of a stoma, preoperative TNM staging, neoadjuvant chemotherapy, curative resection, and LOS. Classification predictive modelling predicted three different colorectal cancer mortality measures (overall mortality, and 31- and 91-days mortality) with 80-96\% accuracy, 84-93\% sensitivity, and $75-100 \%$ specificity. A model using all variables performed only slightly better than one that used just the most significant ones.
\end{abstract}

Presented to the National Cancer Research Institute (NCRI) Virtual Showcase November 2020 and The European Society of Surgical Oncology (ESSO) 2020 Virtual October 2020; published in abstract form as European Journal of Surgical Oncology 2021; 47(Issue 2): e5.

Keywords: Colorectal cancer; Colorectal Surgery; Prediction; Predictor Variables; Length of stay;

Readmission; Mortality; Data Analytics; Artificial Intelligence; Machine Learning 


\section{Introduction}

Colorectal cancer is ranked third on the common cancers list with over 1.8 million new cases in 2018 [9]. Moreover, it is estimated that there will be around 2.4 million cases worldwide in 2035 [17]. Colorectal cancer is the third most common cancer in European populations [25]. The US and the UK have estimated that there will be around 147,950 and 42,300 cases of colorectal cancer respectively in 2020 [48] [54], which is more than 110 in a single day.

LOS, readmission and mortality are essential proxies of quality of care in surgery [4, 43, 46, 52]. Shorter LOS could potentially minimise healthcare costs, free up hospital beds, improve productivity, reduce the risk of nosocomial infections and improve quality of life. Increased readmission rates have a huge impact on healthcare costs. Readmission within 30 days annually costs around 40 billion [20, 24]. Moreover, a higher readmission rate indicates poor discharge planning and post-operative morbidity, with a clinical and psychological impact on the patient. Overall mortality rates following colorectal surgery range from $1 \%$ to $16.4 \%[3,21,51]$. The National Cancer Intelligence Network found that the 30-day post-operative mortality rate is falling across England, with the overall post-operative mortality rate of $6.7 \%$. This rate improved over the study period from $6.9 \%$ in 1998 to $5.9 \%$ in 2006 [38]. The National Bowel Cancer Audit in their recent annual report found a downward trend in 90-day post-operative mortality with a rate of $3.0 \%$. The study also showed that 90-day post-operative mortality has reduced from $2.3 \%$ in $2013 / 14$ to $1.7 \%$ in $2017 / 18$ for elective surgery and from $14.2 \%$ to $11.5 \%$ for emergency surgery [5].

The economic impact of colorectal cancer on healthcare systems is intense. The US is expected to spend around US $\$ 17.41$ billion on colorectal cancer, and approximately US $\$ 4.2$ billion in productivity lost to deaths related to colorectal cancer in 2020 [31]. In the UK, the cost of diagnosing and treating colorectal cancer patients is significant ( $€ 40,000$ per case) [25] and exacerbates funding constraints on the National Health Service (NHS) [4]. With limited resources and a finite surgical bed capacity in many hospitals, it is extremely important to know the expected LoS, readmission rate and mortality after elective CRC surgery.
In recent times, Artificial Intelligence (AI) and Machine Learning (ML) techniques have shown great promise in the diagnosis and prognosis of various diseases and health conditions [27, 39]. ML aims to discover patterns from data without explicit programming. ML algorithms are used to model and learn important properties from data, including the stochastic dependency between a set of input and output variables. ML is a data-driven technique that has the benefit of integrating multiple risk factors into a prediction model [40]. Meanwhile, ML techniques have been found useful in detecting colorectal cancer in advance where the model was constructed with blood cell count, age and sex as input features [23].

An accurate prediction of LOS, readmission and mortality would help healthcare professionals with planning, decision making and building strategies. This will eventually lead to improved patient care and prevent readmission and mortality after discharge [7]. A prediction model that could predict readmission accurately would help healthcare professionals to intervene in readmission scenarios and provide better patient care. A model with the ability to predict the mortality would be valuable to patients, surgeons and healthcare institutions. An accurate mortality prediction model would contribute to patient risk stratification, preoperative consultation with the patient and family members, decision-making process, consent and professional accountability.

This study has investigated the scope of AI and data analytics in predicting LOS, readmission and mortality in colorectal cancer patient's treated in a large NHS trust. Predictor variables of LOS, readmission and mortality have been explored using data analysis to determine which predictors are most important. Machine learning algorithms were then investigated as predictive tools. A comparison has been made between using all variables as predictors for machine learning versus using just the most significant variables.

\section{Methodology}

Codes and findings of feature selection experiments are available in the github repository [32]. The uploaded file in the repository is currently protected with a password, 'colorectalai'. 


\subsection{Data}

Records of a prospectively maintained colorectal cancer database by the Colorectal Department in a large NHS Trust were examined. The dataset contains 4336 patients who underwent colorectal cancer surgery between 2003 and 2019. The 47 patient parameters/variables included demographics, peri- and post-operative outcomes, surgical approaches, complications and mortality (See Table 1). Descriptive statistics of some variables that summarize the central tendency, dispersion and shape of the distribution of a dataset, excluding $\mathrm{NaN}$ values, can be seen in Table 2. There were 2494 male and 1942 female patients in the dataset. Among these 4336 cases, 74\% (3209) were curative, $13.35 \%$ (579) were palliative, and $6.53 \%$ (283) were uncertain. $80 \%$ (3475) of the surgeries performed were elective in comparison to $18 \%$ (782) of emergency. Assistance from the robot was considered for $8.9 \%$ (388) of the cases. The laparoscopic approach was applied to $57.45 \%$ (2491) of the cases, whereas open surgery was used in $35.79 \%$ (1552). The 30 -day readmission rate was $7.4 \%$. Moreover, the 30 and 90 day mortality was $3.39 \%(147)$ and $5.93 \%$ (257) respectively.

Table 1 Available variables in the dataset.

\begin{tabular}{|l|l|l|}
\hline Sex & Complication & \\
ASA & Additional procedures & pN stage \\
Age & Stoma formation & Resection margin \\
BMI & Robotic & Radio therapy \\
Cancer site & Laparoscopic & Chemo therapy \\
TumICD10 & Laparoscopic type & misc_info \\
Preoperative T stage & Curative Surgery & Mortality \\
Preoperative nodal stage & Operation time & Death_date \\
Preoperative M stage & Blood loss & Death_check \\
Previous abdominal surgery & LOS & Private_pt \\
Previous surgery type & Readmit $<31$ days & Local recurrence date \\
Operation mode & REOP $<31$ days & Distant recurrence date \\
Resection $(y / n)$ & Complication misc & Distant recurrence days \\
Procedure type $(4$ classes $)$ & LN harvest & Local recurrence days \\
OPCS4 & LN positive & Mortality $<31$ days \\
Specific procedure $(18$ classes $)$ & pT stage & Mortality $<91$ days \\
\hline
\end{tabular}

Table 2 Statistics of selected variables from the dataset.

\begin{tabular}{|c|c|c|c|c|c|c|}
\hline $\begin{array}{c}\text { Descriptive } \\
\text { Statistics }\end{array}$ & Age & $\begin{array}{c}\text { Operation } \\
\text { time }\end{array}$ & $\begin{array}{c}\text { Blood } \\
\text { loss }\end{array}$ & BMI & LOS & $\begin{array}{c}\text { Mortality } \\
\text { days }\end{array}$ \\
\hline Mean/Average & 70.24 & 181.18 & 66.16 & 26.85 & 11.26 & 1131.32 \\
\hline Standard Deviation & 11.61 & 93.15 & 78.89 & 4.26 & 12.12 & 1152.21 \\
\hline Minimum value & 24.00 & 0.00 & 0.00 & 13.50 & 0.00 & 0.00 \\
\hline 25th Percentile & 63.00 & 160.00 & 50.00 & 25.00 & 5.00 & 245.25 \\
\hline 50th Percentile & 72.00 & 181.18 & 66.16 & 26.85 & 8.00 & 724.50 \\
\hline 75th Percentile & 79.00 & 215.00 & 66.16 & 28.00 & 13.00 & 1654.00 \\
\hline Maximum Value & 97.00 & 690.00 & 1200.00 & 78.50 & 252.00 & 5542.00 \\
\hline
\end{tabular}

\subsubsection{Data Processing}

The problems of missing values and mixed data types were dealt with using appropriate techniques and with the help of medical domain knowledge through discussions with clinicians. Following clinical discussion, missing values were filled with different techniques (see Table 3). The dataset consists of some columns where data types are mixed. For example, Sex and TumICD10 variables have both text and numeric data. In order to fit them to machine learning algorithms, all these mixed data types are converted to numeric data using the Pandas Series.str.replace() method [35]. Moreover, there are some columns that comprise text values only (e.g., Robotic, Radiotherapy). All these columns that consist of text data are passed through the LabelEncoder methods of scikit learn [41] to convert them to numeric data.

Table 3 Alternative techniques to fill missing values with medical domain knowledge.

\begin{tabular}{|c|c|c|}
\hline Variables & No of Missing Values & Methods to fill missing values \\
\hline Sex & 0 & - \\
\hline ASA & 593 & Mode \\
\hline LOS & 179 & Mean \\
\hline Operation Mode & 79 & Min \\
\hline Curative Surgery & 265 & Min \\
\hline
\end{tabular}

\subsection{Prediction Model Building}

\subsubsection{Model for LOS}

Regression predictive modelling is performed to predict the LOS. The data are power-transformed to make them more Gaussian-like [61]. Then the data are discretized to map numerical variables onto discrete values. Such mapping creates a highorder ranking of values that can smooth out the relationships between observations and is found useful for machine learning [28]. 10-fold crossvalidation techniques $[36,44]$ are used for splitting the training and test data. Different algorithms were compared to find the optimal model for LOS prediction. A negative mean absolute error was used as the evaluation metric to compare different algorithms. Comparison between algorithms shows that support vector regression (SVR) outperformed the other algorithms (see Figure 1). Following this finding, different parameters of the SVR algorithms (see Table 4) were tuned using the GridSearchCV technique [8]. The model was 
trained with the training dataset and tested on the test dataset. Finally, different evaluation metrics, namely root-mean-square error (RMSE), mean absolute error (MAE), and accuracy, were used to evaluate the model. Data analysis of different variables in predicting LOS was also conducted.

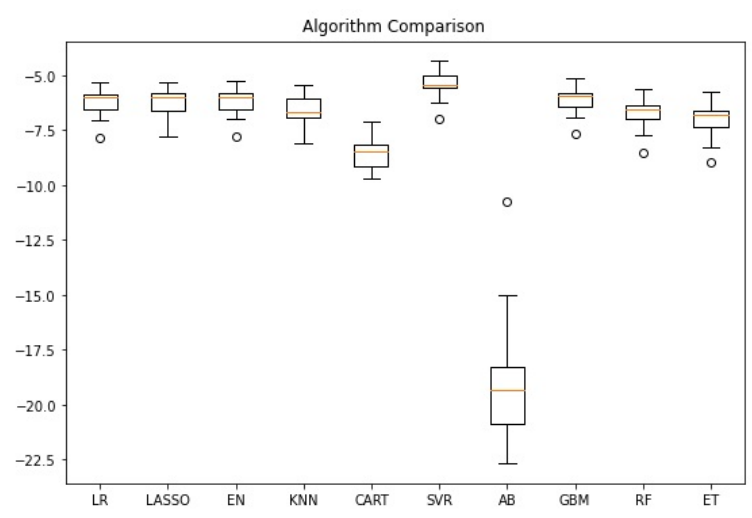

Fig. 1 Comparison of algorithms for modelling the data.

Table 4 Tuning the parameters of the SVR algorithm.

\begin{tabular}{|c|c|c|}
\hline Parameters & Range & Best Parameters \\
\hline kernel & ['linear', 'poly', 'rbf'] & rbf \\
\hline $\mathrm{C}$ & {$[1,5,10,15]$} & 10 \\
\hline degree & {$[1,2,3]$} & 1 \\
\hline gamma & {$[$ 'scale', 'auto'] } & scale \\
\hline coef0 & {$[0, .01, .1]$} & .01 \\
\hline epsilon & {$[.1, .5, .9]$} & .9 \\
\hline
\end{tabular}

\subsubsection{Model for Readmission}

Classification predictive modelling is performed to predict the readmission. Models with Random Forest (RF), K Nearest Neighbor (KNN), Support Vector Machine (SVM), Multilayer Perceptron (MLP), and Bidirectional Long Short-Term Memory (BI-LSTM) algorithms were compared for readmission prediction. For the BI-LSTM algorithm, the data are reshaped following the work of Masum et al $[33,34]$ as the LSTM based RNN requires input to be in a matrix with the dimensions: [samples, time steps, features]. The model with BI-LSTM algorithm has been designed so that the network structure consists of three hidden layers with 100 LSTM units, then an output layer with the sigmoid activation. The network also represented binary crossentropy as a loss function,
ADAM algorithm [26] as an optimizer, and accuracy as metrics. The network has been fitted with 20 epochs and a batch size of $2.80 \%$ of data of the dataset was used for training and $20 \%$ was used for testing purpose.

\subsubsection{Model for Mortality}

Classification predictive modelling is performed to predict the mortality. Models with Random Forest (RF), K Nearest Neighbor (KNN), Support Vector Machine (SVM), Multilayer Perceptron (MLP), and Bidirectional Long Short-Term Memory (BILSTM) algorithms were compared for mortality, and 31- and 91-days mortality prediction. The model structure for readmission prediction mentioned in section 2.2.2 was used for mortality prediction scenarios.

\subsection{Comparing variables}

The variable that needs to be predicted is known as the target variable and the variables that are used to predict the target variable known as features. Identifying the best features is an important task [19]. A large number of features could lead to complex model, long training time, the curse of dimensionality, noise addition, overfitting etc. On the other hand, a smaller number of variables could lead to the exclusion of relevant variables. ExtraTreeRegressor [18], ExtraTreesClassifier [18], LassoCV [53] and Correlation Matrix analysis with Heat Map of scikit-learn [41] have been considered for feature selection. Moreover, in all prediction cases, $80 \%$ of the dataset was used for training and $20 \%$ was used for testing purposes.

\subsubsection{Feature Selection for LOS}

Extra Tree Regressor showed that Age, BMI, Surgical approach, Operation time, ASA, Blood loss, Preoperative T stage, Stoma formation, Sex and Preoperative nodal stage were the most crucial features in predicting LOS (see github repository [32]). In contrast, a LASSO algorithm showed that Surgical approach, Sex, Chemotherapy, ASA, Operation mode, Stoma formation, TumID10, Procedure type, Additional procedures, Radiotherapy, Preoperative T stage, Age and, Cancer site were the most important features 
(see github repository [32]). Moreover, the features explored through a correlation matrix with heat map has found Surgical approach, ASA, Age, Operation mode, Complication, Stoma formation, Chemotherapy, TumID10, Preoperative $\mathrm{T}$ stage as essential features (see github repository [32]). Following the findings from these techniques, we considered Age, ASA, Surgical approach, Stoma formation, Preoperative T stage, Chemotherapy, Operation mode, TumID10, Cancer site, and Radiotherapy as the selected features for predicting LOS.

\subsubsection{Feature Selection for Readmission}

Extra Tree Classifier showed that Surgical approach, Operation time, LOS, BMI, Age, ASA, Blood loss, Preoperative T stage, Stoma formation were the most crucial features in predicting readmission (see github repository [32]). In contrast, a LASSO algorithm showed that Surgical approach, Operation mode, Previous surgery type, Stoma formation, Preoperative nodal stage, the Specific procedure, ASA, BMI, Age, Sex, LOS and Cancer site were the most important features (see github repository [32]). Moreover, features explored through a correlation matrix with heat map have found Surgical approach, Age, Preoperative nodal stage, Pre abdominal surgery, Previous surgery type, Operation mode, Complication, Additional procedures, Robotic, Curative Surgery, Operation time and LOS as essential features (see github repository [32]). Following the findings from these techniques, we considered Age, Surgical approach, Stoma formation, Preoperative nodal stage, Operation time, Operation mode, Previous surgery type, LOS, and the Specific procedure as the selected features for predicting readmission.

\subsubsection{Feature Selection for Mortality}

Extra Tree Classifier showed that Age, LOS, Curative Surgery, BMI, ASA, Operation time, Surgical approach, Chemotherapy, Radiotherapy, Operation mode were the most critical features in predicting mortality (see github repository [32]). A LASSO algorithm showed that Curative Surgery, Chemotherapy, Preoperative T stage, Operation mode, ASA, Preoperative M stage, Stoma formation, Age, the Specific procedure, LOS, OPCS4,
BMI, Cancer site, Previous surgery type, and Surgical approach were the most important features (see github repository [32]). Moreover, features explored through a correlation matrix with heat map has found ASA, Age, BMI, Preoperative T stage, Preoperative $\mathrm{M}$ stage, Operation mode, Procedure type, Complication, Stoma formation, Radiotherapy, Chemotherapy, Surgical approach, Curative Surgery and LOS as essential features (see github repository [32]). Following the findings from these techniques, we considered Age, ASA, BMI, Chemotherapy, Preoperative M stage, Surgical approach, LOS, Curative Surgery, Preoperative $\mathrm{T}$ stage and Stoma formation as the selected features for predicting mortality. For 31 days mortality prediction, we selected Chemotherapy, Additional procedures, Operation mode, Complication, Previous abdominal surgery, Previous surgery type, Surgical approach, Curative Surgery, Age and Resection as selected features. In contrast, for 91 days mortality prediction we selected LOS, Additional procedures, Curative Surgery, Complication, Previous surgery type, Pre abdominal surgery, Procedure type, Operation mode, Surgical approach and Resection as the selected features.

\subsubsection{Model for comparing variables in LOS, readmission and mortality prediction}

The model with the SVR algorithm mentioned in section 2.2.1 was used in comparing variables in LOS prediction. To compare variables in readmission prediction, the model with BILSTM algorithm mentioned in section 2.2.2 was used. Moreover, the model with BI-LSTM algorithm used in section 2.2.3 was used in comparing variables in all scenarios of mortality prediction.

\section{Results}

\subsection{LOS Prediction}

The model with a SVR algorithm predicted the LOS with an MAE and RMSE of 9.69 and 12.52 respectively. Figure 2 shows how the model predicted the LOS. The regression outcome of the model is then converted to binary class using these conditions: 


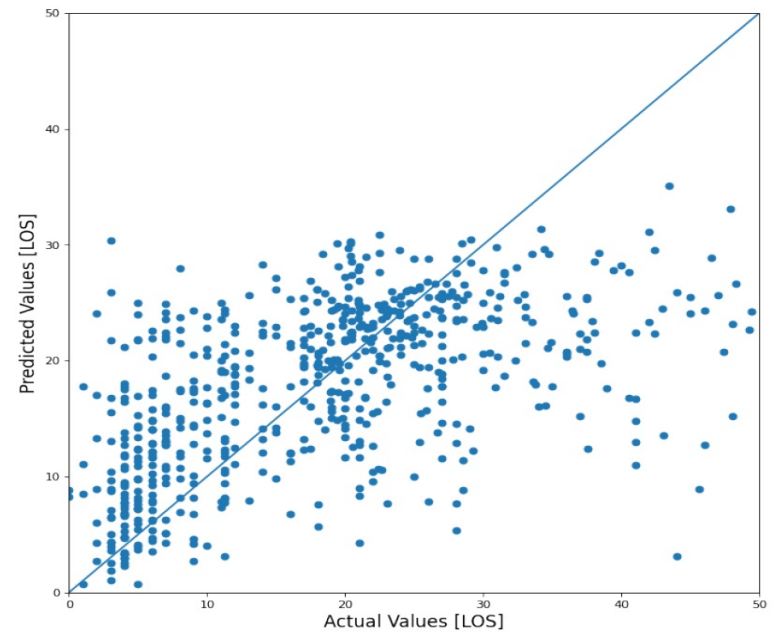

(a)

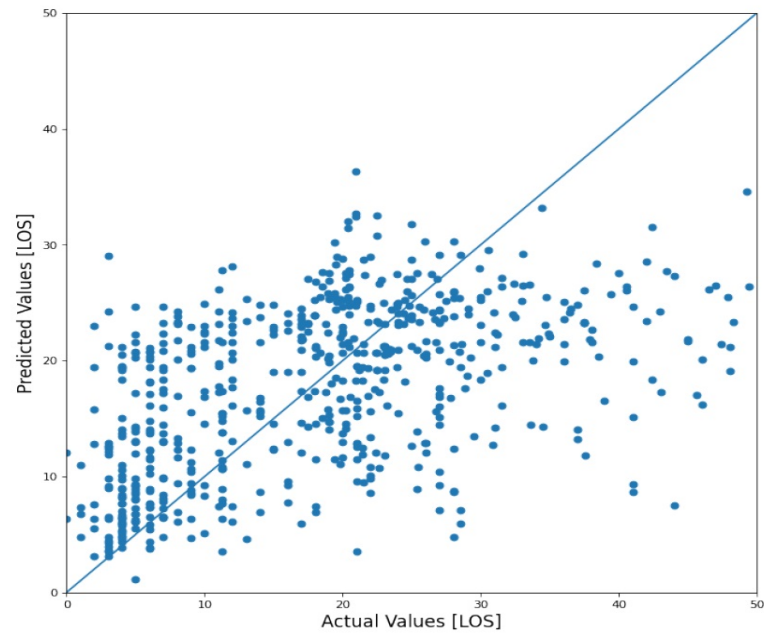

(b)

Fig. 2 LOS prediction: the figure (a) represents the actual versus predicted LOS when considering all variables and the figure (b) represents the actual versus predicted LOS when considering only selected variables.

- True class $(1)=$ if patient's stay is correctly predicted $\leq 6$ days

- True class $(1)=$ if patient's stay is correctly predicted $>6$ days

- False class $(0)=$ if patient's stay is incorrectly predicted $\leq 6$ days

- False class $(0)=$ if patient's stay is incorrectly predicted $>6$ days

Converting the regression outcome to a binary class helps to calculate the accuracy of the model and accuracy of $83.21 \%$ was recorded when all available variables were considered as inputs.

\subsection{Data analysis of LOS}

Data analysis of different variables for LOS shows that age groups, fitness, whether a robotic surgery was performed or not, whether a laparoscopic operation was performed or not, operation mode and complications all have a significant impact in LOS prediction. LOS was grouped into three categories ( $\leq 6,7-14$ and $\geq 15$ days). Different variables were also categorised accordingly (See Figure 3 ). Data analysis of different variables in relation to LOS indicates that:

- The number of patients who had a LOS of 15 days and over increased with age. Moreover, the number of patients who had a LOS of 6 days and less decreased with age.
- Patients with higher fitness levels (healthier patients) have shorter hospital stays compared with patients with poor fitness levels.

- Observation of the surgical approach shows that a laparoscopic operation leads to shorter LOS compared with open operation.

- Patients with an emergency operation were more likely to have an increased LOS compared with an elective operation.

- Patients with a complication were more likely to have an increased LOS compared with patients without difficulty.

- Patients' stays in hospital are shorter when robotic surgery was performed in comparison with non-robotic surgery.

- It was also observed that BMI is not a good indicator of LOS.

\subsection{Readmission Prediction}

The dataset contains 321 readmission cases. Different algorithms were compared in readmission prediction, and it was found that the model with a BI-LSTM algorithm outperformed the other algorithms (see Table 5). The model with a BILSTM algorithm predicted the readmission with an accuracy of $87.5 \%$, a sensitivity of $84.1 \%$ and a specificity of $90.9 \%$. ROC curve of the model for predicting readmission is presented in Figure 4. 

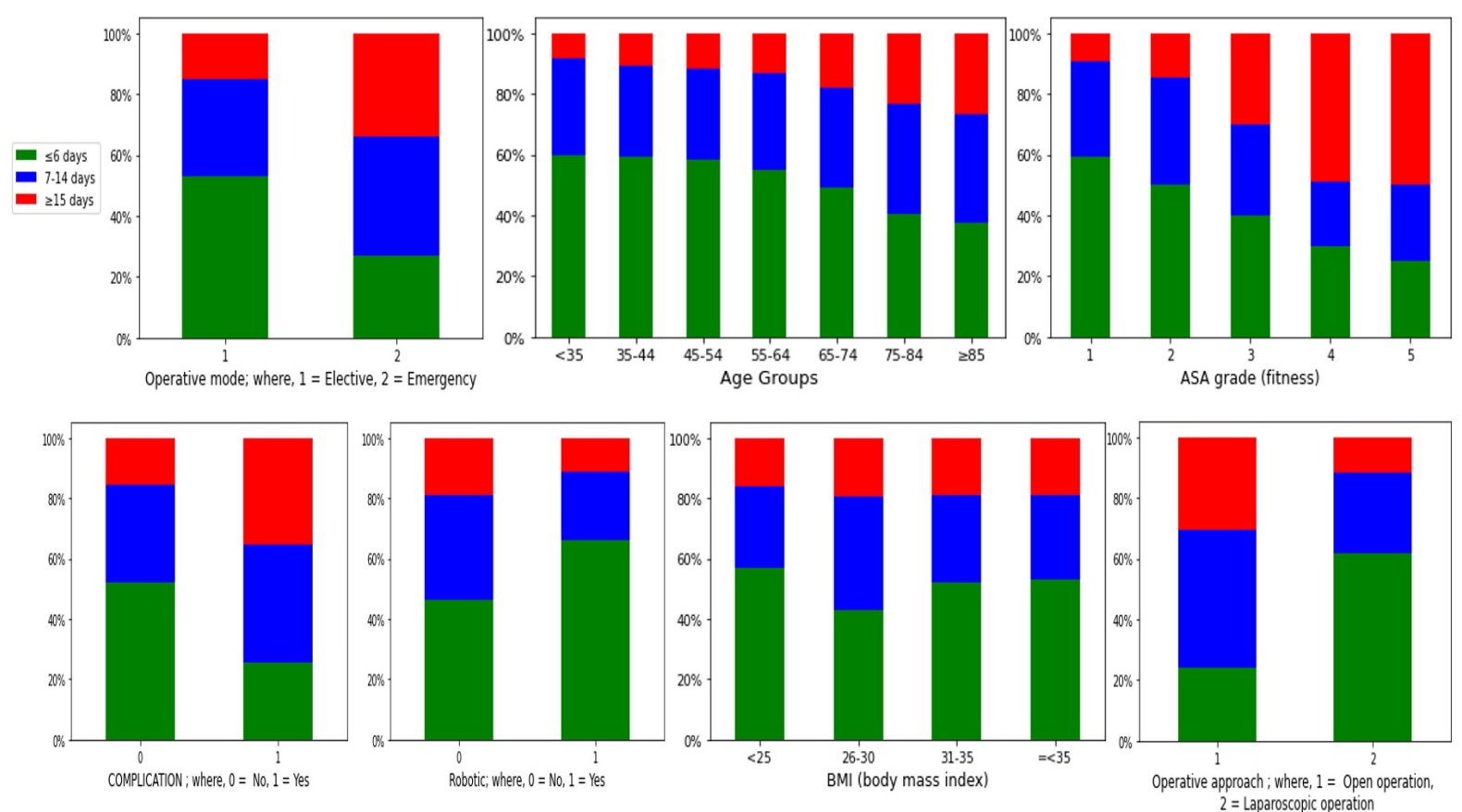

Fig. 3 Data analysis of LOS.

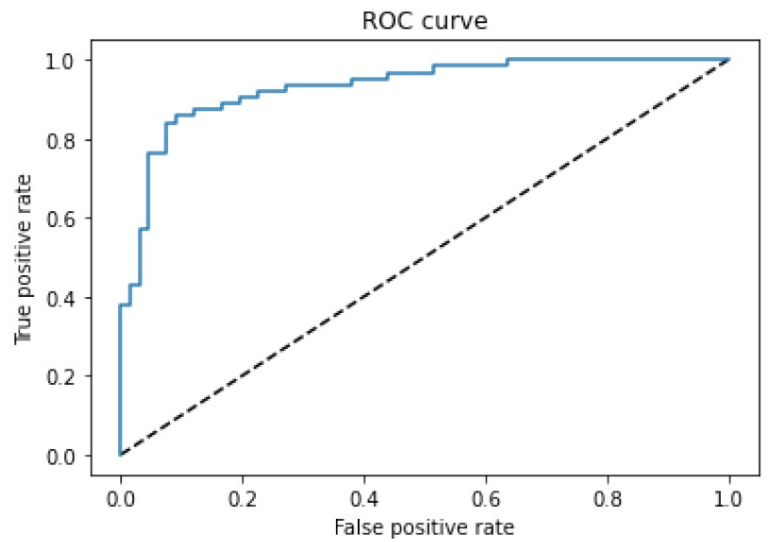

(a)

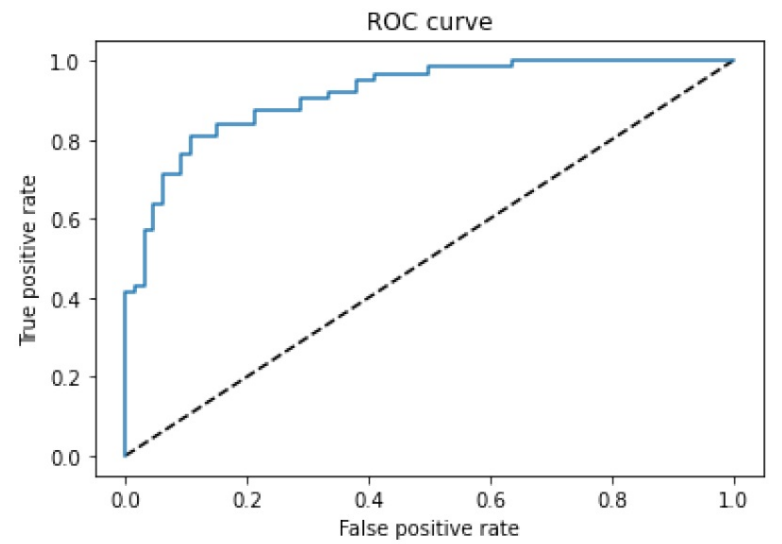

(b)

Fig. 4 Readmission prediction: the figure (a) represents the ROC CURVE when considering all variables and the figure (b) represents the ROC CURVE when considering only selected variables.

Table 5 Prediction of readmission with different algorithms.

\begin{tabular}{|c|c|c|c|}
\hline Algorithm & Accuracy & Sensitivity & Specificity \\
\hline Random Forest & .768 & .753 & .781 \\
\hline KNN & .675 & .619 & .728 \\
\hline SVM & .681 & .482 & .878 \\
\hline MLP & .740 & .725 & .757 \\
\hline BILSTM & .875 & .841 & .909 \\
\hline
\end{tabular}

\subsection{Data analysis of Readmission}

Data analysis of different variables for readmission shows that the following factors have a strong impact on readmission: fitness, operation mode, additional procedure, whether the cancer is curative or not, previous abdominal surgery, types of previous surgery, preoperative $M$ stage and time of the operation. Readmission was grouped into 

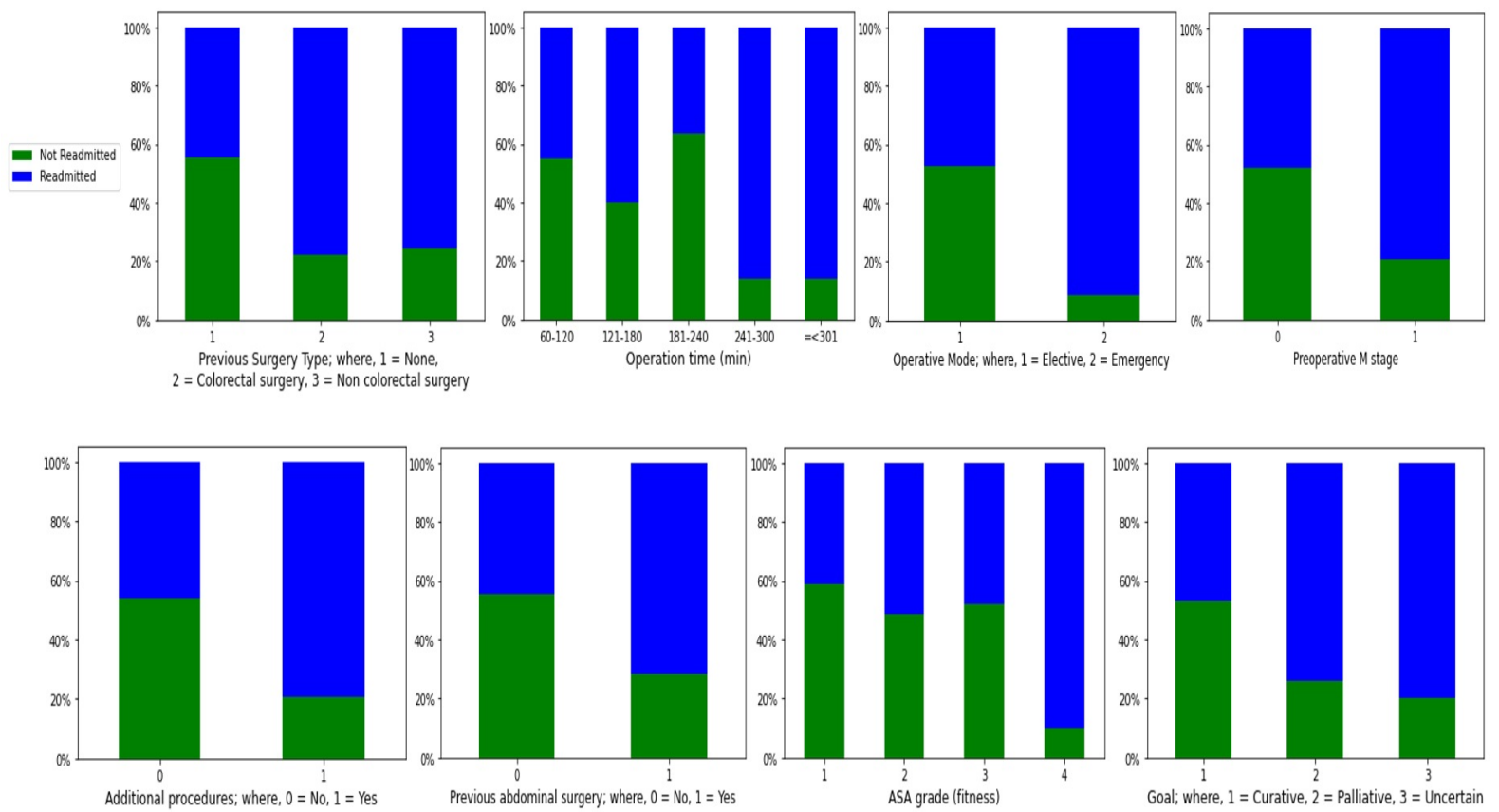

Fig. 5 Data analysis of Readmission.

two categories (Readmitted and Not Readmitted). Different variables were also categorised accordingly (See Figure 5). Data analysis of different variables concerning readmission indicates that:

- A higher percentage of patients are readmitted in a palliative and uncertain scenario compared with a curative scenario.

- Patients with an emergency operation are more likely to be readmitted than those with an elective operation.

- Additional procedures during surgery increase the chance of readmission.

- The readmission rate following colorectal surgery is low for patients with better performance status (ASA grade) compared with poor performance status (ASA grade).

- Previous abdominal surgery before colorectal surgery increases the readmission rate.

- Previous surgery (both bowel and non-chronic groups) prior to colorectal surgery also increases the readmission rate.

- Patients with a preoperative M stage have a high chance of getting readmitted compared with those without.

- A longer operation time increases the patients' likelihood of readmission.

\subsection{Mortality Prediction}

The model with BI-LSTM algorithms predicted the overall mortality with an accuracy of $80 \%$, a sensitivity of $84.3 \%$ and specificity of $75.3 \%$. The BI-LSTM algorithms outperformed random forest, KNN, SVM and MLP in predicting mortality prediction. The model with a random forest algorithm predicted the 31 days mortality with an accuracy of $98.9 \%$, a sensitivity of $97.9 \%$ and a specificity of $100 \%$. The model with BI-LSTM algorithm also performed well in predicting 31 days mortality with an accuracy of $96.6 \%$, a sensitivity of $93.7 \%$ and a specificity of $100 \%$. Moreover, the dataset only contains 146 cases of 31 days of mortality.

The model with a BI-LSTM algorithm predicted the 91 days mortality with an accuracy of $94.2 \%$, a sensitivity of $91.4 \%$ and a specificity of $96.3 \%$. The model with a random forest algorithm performed second-best in predicting 91 days mortality with an accuracy of $87.9 \%$, a sensitivity of $89.4 \%$ and a specificity of $86.4 \%$. ROC curve of the model predicting 91 days mortality is presented in Figure 6 . The dataset contains only 257 cases of 91 days of mortality. 


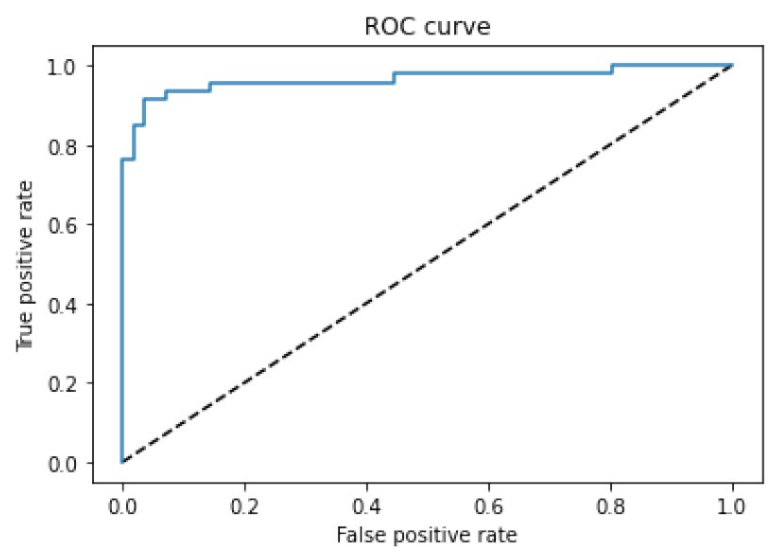

(a)

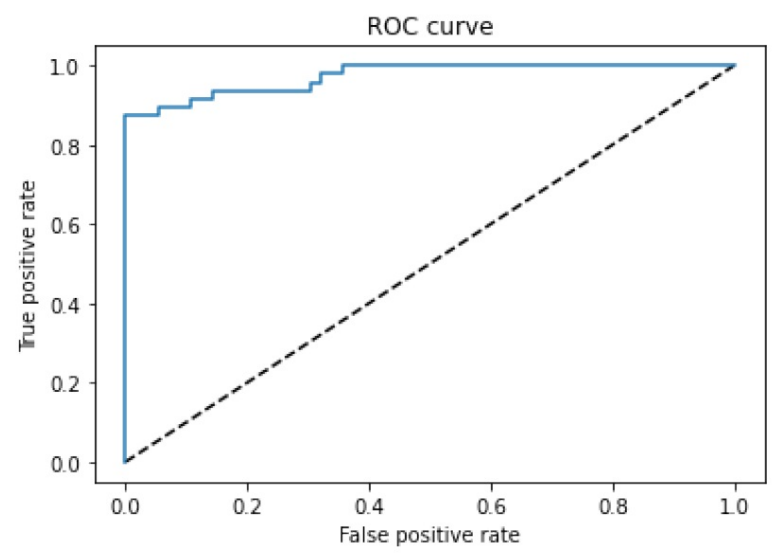

(b)

Fig. 6 Prediction of 91 days mortality: the figure (a) represents the ROC CURVE when considering all variables and the figure (b) represents the ROC CURVE when considering only selected variables.

Table 6 Predictions of mortality, 31 days mortality, and 91 days mortality with different algorithms.

\begin{tabular}{|c|c|c|c|c|l|l|l|l|l|}
\hline & \multicolumn{3}{|c|}{ Mortality } & \multicolumn{3}{c|}{ 31 Days Mortality } & \multicolumn{3}{c|}{ 91 Days Mortality } \\
\hline Algorithms & Accuracy & Sensitivity & Specificity & Accuracy & Sensitivity & Specificity & Accuracy & Sensitivity & Specificity \\
\hline Random Forest & .701 & .590 & .812 & .989 & .979 & 1 & .879 & .894 & .864 \\
\hline KNN & .657 & .595 & .719 & .818 & .877 & .760 & .798 & .852 & .744 \\
\hline SVM & .635 & .679 & .590 & .760 & .918 & .699 & .743 & .898 & .589 \\
\hline MLP & .740 & .778 & .757 & .900 & .911 & .910 & .852 & .871 & .833 \\
\hline BILSTM & .800 & .843 & .753 & .966 & .937 & 1 & .942 & .914 & .963 \\
\hline
\end{tabular}

\subsection{Data analysis of Mortality days}

Data analysis of mortality days shows that fitness, complication, additional procedures, operation mode, whether the cancer is curative or not and preoperative $\mathrm{M}$ stage have a strong impact on mortality days. Mortality days were grouped into five categories ( $\leq 90,91-180,181-365,366-730$ and $>730$ days). Different variables were also categorised accordingly (See Figure 7). Data analysis of different variables concerning mortality days indicates that:

- Patients with better performance status (ASA grade) live longer than those with poor performance status (ASA grade) following colorectal surgery.

- Complications during colorectal surgery lead to shorter lives compared with no complications. Postoperative complications also have a direct impact on patient survival.

- Patients with a palliative scenario would live for a shorter period compared with a curative and uncertain scenario.
- Patients with preoperative M1 stage have a reduced life expectancy compared with those without.

- Additional procedures during index operation indicate advanced stage of disease and are associated with prolonged operative time and reduced survival.

- Patients with elective surgery would live longer compared with emergency surgery.

- It was also observed that BMI is not a good indicator of mortality.

\subsection{Comparison between all variables and selected variables}

The model with the SVR algorithm mentioned in section 2.3.4 was fed with two different types of the dataset to predict the LOS. In one case, all 27 relevant features related to LOS were used as input. In contrast, in the other case, 11 selected variables mentioned in section 2.3.1 were used as the input in predicting LOS. Comparison between the two scenarios can be seen in Table 7. It shows that the model with all variables as features performs only slightly better than the model with selected variables. The model that used all variables as features 

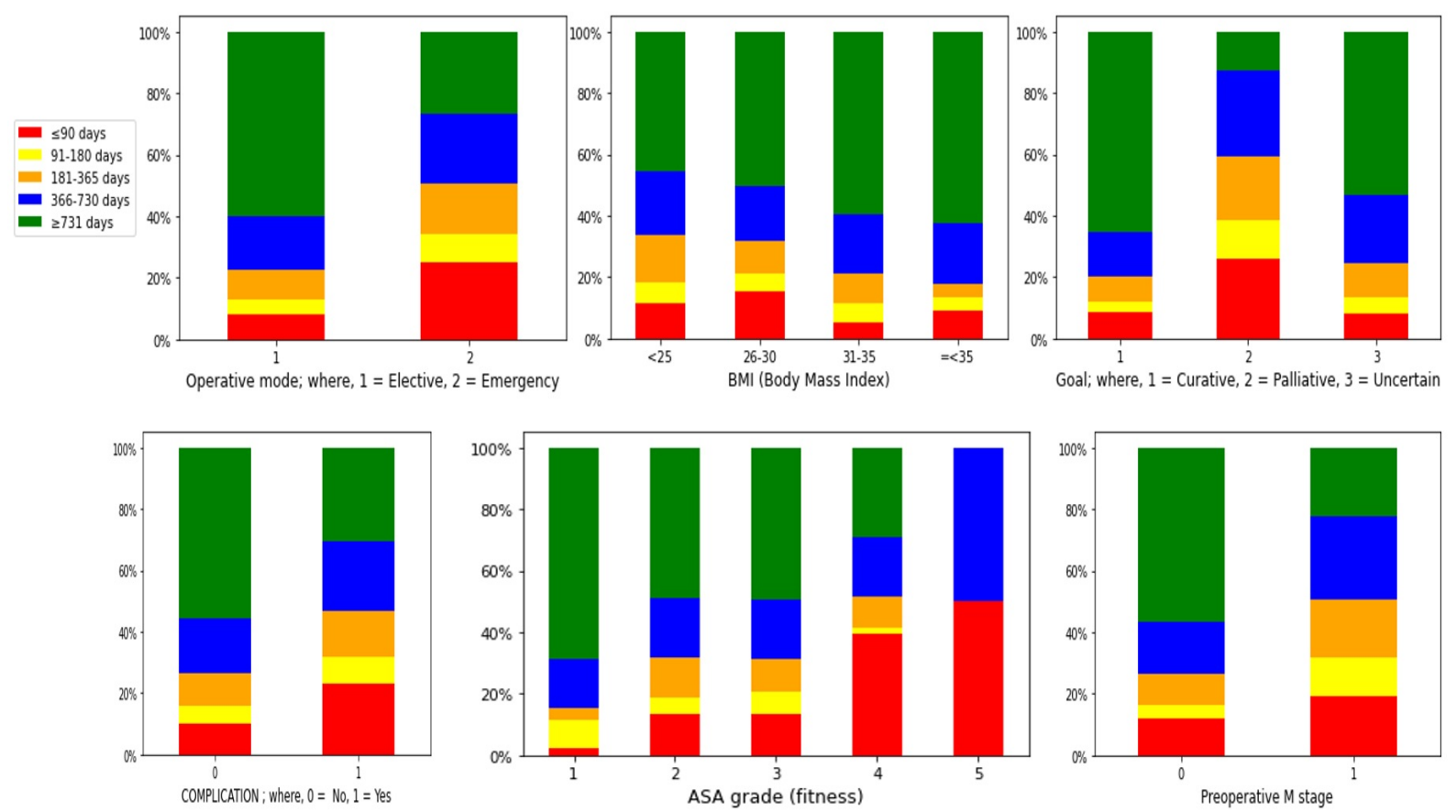

Fig. 7 Data analysis of mortality days.

predicted the LOS with an accuracy of $83.21 \%$, MAE of 9.69 and RMSE of 12.52. In contrast, an accuracy of $82.61 \%$, MAE of 10.32 and RMSE of 12.79 were recorded when the model used selected variables. Comparison of actual versus predicted LOS of both cases can be seen in Figure 2 .

Comparison between all variables and selected variables in predicting readmission can be seen in Table 8. It shows that the model mentioned in section 2.3.4 with the use of all 28 variables predicted the readmission scenario with an accuracy of $87.5 \%$, a sensitivity of $84.1 \%$ and specificity of $90.9 \%$. This outperforms the model outcome where nine selected variables were considered. The model with selected variables predicted the readmission scenario with an accuracy of $83.7 \%$, a sensitivity of $76.1 \%$ and specificity of $90.9 \%$. ROC curve of each scenario is also presented in Figure 4.

The model mentioned in section 2.3.4 for mortality prediction predicted the mortality with an accuracy of $80 \%$, a sensitivity of $84.3 \%$ and specificity of $75.3 \%$ when all 29 variables were considered as features. The model performance decreased when the 10 selected variables were considered. An accuracy of $78.4 \%$, sensitivity of $79 \%$ and specificity of $77.8 \%$ were recorded (see Table 9
). The model performance does not vary much for 31 and 91 days mortality when these two different sets of data were used for comparison (see Tables 10 and 11). ROC curve of each scenario predicting 91 days mortality is also presented in Figure 6.

Table 7 Comparison between the use of all variables versus selected variables in LOS prediction.

\begin{tabular}{|c|c|c|}
\hline Evaluation Metrics & $\begin{array}{c}\text { All variables as feature } \\
(27)\end{array}$ & $\begin{array}{c}\text { Selected variables as feature } \\
(\mathbf{1 0})\end{array}$ \\
\hline RMSE & $\mathbf{1 2 . 5 2}$ & $\mathbf{1 2 . 7 9}$ \\
\hline MAE & $\mathbf{9 . 6 9}$ & $\mathbf{1 0 . 3 2}$ \\
\hline Accuracy & $\mathbf{8 3 . 2 1}$ & $\mathbf{8 2 . 6 1}$ \\
\hline
\end{tabular}

Table 8 Comparison between the use of all variables versus selected variables in readmission prediction.

\begin{tabular}{|c|c|c|}
\hline Evaluation Metrics & $\begin{array}{c}\text { All variables as feature } \\
(28)\end{array}$ & $\begin{array}{c}\text { Selected variables as feature } \\
(9)\end{array}$ \\
\hline Accuracy & $\mathbf{8 7 . 5}$ & $\mathbf{8 3 . 7}$ \\
\hline Sensitivity & $\mathbf{8 4 . 1}$ & $\mathbf{7 6 . 1}$ \\
\hline Specificity & $\mathbf{9 0 . 9}$ & $\mathbf{9 0 . 9}$ \\
\hline
\end{tabular}

Table 9 Comparison between the use of all variables versus selected variables in mortality prediction.

\begin{tabular}{|c|c|c|}
\hline Evaluation Metrics & $\begin{array}{c}\text { All variables as feature } \\
(29)\end{array}$ & $\begin{array}{c}\text { Selected variables as feature } \\
(\mathbf{1 0})\end{array}$ \\
\hline Accuracy & $\mathbf{8 0 . 0}$ & $\mathbf{7 8 . 4}$ \\
\hline Sensitivity & $\mathbf{8 4 . 3}$ & $\mathbf{7 9 . 0}$ \\
\hline Specificity & $\mathbf{7 5 . 3}$ & $\mathbf{7 7 . 8}$ \\
\hline
\end{tabular}


Table 10 Comparison between the use of all variables versus selected variables in 31 days mortality prediction.

\begin{tabular}{|c|c|c|}
\hline Evaluation Metrics & $\begin{array}{c}\text { All variables as feature } \\
(29)\end{array}$ & $\begin{array}{c}\text { Selected variables as feature } \\
(10)\end{array}$ \\
\hline Accuracy & 96.6 & 96.6 \\
\hline Sensitivity & 93.7 & 93.9 \\
\hline Specificity & 100.0 & 100.0 \\
\hline
\end{tabular}

Table 11 Comparison between all variables versus selected variables in 91 days mortality prediction.

\begin{tabular}{|c|c|c|}
\hline Evaluation Metrics & $\begin{array}{c}\text { All variables as feature } \\
(29)\end{array}$ & $\begin{array}{c}\text { Selected variables as feature } \\
(10)\end{array}$ \\
\hline Accuracy & $\mathbf{9 4 . 2}$ & $\mathbf{9 4 . 1}$ \\
\hline Sensitivity & 91.4 & $\mathbf{8 7 . 2}$ \\
\hline Specificity & $\mathbf{9 6 . 3}$ & $\mathbf{1 0 0 . 0}$ \\
\hline
\end{tabular}

\section{Discussion}

\subsection{Role of data analytics and machine learning}

LOS, readmission and mortality are widely used proxies and quality indicators of care and healthcare spending following colorectal surgery. Accurate prediction of these three proxies remains a crucial challenge after colorectal cancer surgery and would lead to substantial resource implications for clinical and management teams. Consequently, this study has aimed to predict LOS, readmission, and mortality with various machinelearning algorithms. Moreover, different predictor variables were explored and investigated through data analysis and machine learning techniques. A single centre's data were used for the experiments. The dataset was then processed according to the models' requirements. Data analysis and feature analysis of the dataset were also performed.

Prior research has used a limited number of variables to investigate LOS, mortality and readmission following CRC surgery $[1,4,25,37,43$, $46,57,58]$. This study includes a larger number of variables (47) including demographics, periand post-operative outcomes, surgical approaches, complications and mortality. These variables are used and compared in predicting LOS, mortality and readmission. Sets of key variables were identified using various data analysis techniques. Algorithms like Extra Tree Regressor, LASOO and correlation matrix with heat map help to extract essential features from all variables. Comparison between using all variables and selected variables has shown that the machine learning model performs better with all variables than the selected variables in predicting LOS, readmission and mortality prediction. This observation confirms the benefit of applying machine learning algorithms when high-dimensional data are available. In principle, a ML algorithm ought to be able to make use of all available information, giving a lower weighting to the less useful information.

Medical researchers have found ML algorithms helpful to predict the diagnosis and prognosis of various diseases and health conditions accurately $[6,14,27,39]$. Moreover, a prediction model with a machine learning algorithm was used to detect early colorectal cancer and recurrence of stage IV colorectal cancer after tumour resection [23, 60]. However, prior studies related to patient LOS, readmission and mortality after colorectal surgery have been mainly limited to observation study of predictor variables, with little investigation of machine learning. The current study explores not only the significant predictor variables, but also investigates machine learning algorithms that can exploit them.

\subsection{LOS prediction}

Pucciarelli et al., on their observational analysis, found median LOS is 13 days [43] in comparison to 11.26 days in this study. Kelly et al., in their research, found median LOS is 14 days for elective and 21 for emergency admissions [25]. Aravani et al. found that age, comorbidity, socioeconomic deprivation, stage of the disease, and emergency operations are better predictor variables of longer LOS [4]. Age, comorbidities, marital status and emergency readmission were linked to the likelihood of longer LOS [25]. Ahmed et al. showed that ASA grade, epidurals and oral opiates are associated with an earlier discharge [1]. Chiu et al. state that minor and major complications were better predictors of LOS than preoperative demographic and disease parameters [10]. Sex, congestive heart failure, weight loss, Crohn's disease, preoperative albumin $<3.5 \mathrm{~g} / \mathrm{dL}$ and hematocrit $<47 \%$, baseline sepsis, ASA class $\geq 3$, open surgery, surgical time $\geq 190 \mathrm{~min}$, post-operative pneumonia, failure to wean from mechanical ventilation, deep venous thrombosis, urinary tract infection, systemic sepsis, surgical site infection and reoperation within 30-days from the primary surgery were the risk factors for prolonged LOS [30]. In contrast, this study found age, ASA grade, operative time, presence or absence of a stoma, robotic or laparoscopic 
approach to surgery, and complications are the significant predictor variables of LOS.

This study investigated the predictor variables' scope in predicting LOS by building a prediction model with machine learning techniques, unlike the prior work. For LOS prediction, models were built using different machine learning algorithms, and the results were compared to find the best model. The model with SVR algorithms turns out to be the best and tuned further with a tuning algorithm. Finally, the adjusted model is used for LOS prediction, and the model predicted the LOS with an MAE and RMSE of 9.69 and 12.52, respectively. The LOS prediction regression outcome is then converted to a binary class with a few conditions mentioned in section 3.1, which showed the model could predict LOS with $83.21 \%$ accuracy. Data analysis of different predictor variables of LOS shows that Age groups, ASA grade, Surgical approach, Operation mode, Complication and Robotic surgery are the most important predictor variables for LOS Prediction. On the other hand, the data analysis also shows that BMI is not a good predictor for LOS prediction.

\subsection{Readmission prediction}

A national population-based study by Pucciarelli et al. found that gender, hospital location, comorbidities, type of surgery, stoma creation, open approach, rectal tumour location, and longer LOS were the predictor variables of 30-day readmission [43]. Chung et al. found that surgical site infection, hepatic disease, pulmonary disease, TNM stage, and operation time were the significant risk factors for readmission [11]. In contrast, this study found that Age, Surgical approach, Stoma formation, Preoperative nodal stage, Operation time, Operation mode, Previous surgery type, LOS, and the Specific procedure were the significant predictor variables for readmission.

A recent study by Rubens et al. created a risk model for predicting 30-day readmission rates after surgical treatment for colon cancer. Their model showed $60.2 \%$ accuracy, $58.8 \%$ sensitivity and $60.4 \%$ specificity with a limited number of variables [45]. In contrast, this study showed that the BI-LSTM algorithm predicts the readmission with an accuracy, sensitivity and specificity of $87.5 \%, 84.1 \%$ and $90.9 \%$, respectively. Data analysis of the dataset found a 30-day readmission rate of $7.4 \%$, which is comparable with the two recent reviews and meta-analyses findings ranged between $7 \%$ and $25 \%$ [13, 29].

\subsection{Post-operative mortality prediction}

The risk of post-operative mortality after CRC has been investigated through several scoring systems $[2,12,15,42,49,50]$. To date, none of these scoring systems has been found effective as a predictor, and researchers have raised questions over their accuracy and usability $[16,37,47,55]$. Moreover, these scoring systems require a high level of preoperative information, including laboratory values which may not always be available and thus they have not been employed widely [59]. Murray et al. found that age, $\mathrm{ASA} \geq 3$, renal failure, ascites, heart failure, disseminated cancer, hypoalbuminemia, open surgery, non-independent status and admission from a chronic care facility are the risk factors of 30-day mortality [37]. Wilkins et al. found that age, ASA grade IV-V, Dukes' stage D, and urgent surgery are strongly associated with post-operative mortality. Their model predicted mortality with an area under the curve of 0.88 [58].

In contrast, this study considered postoperative mortality as a simple binary classification scenario. Prediction models were built and compared with machine-learning algorithms to predict mortality, 31 and 91 days mortality. Moreover, well-known evaluation metrics (i.e., accuracy, sensitivity and specificity) were used to evaluate the model performance. Machine learning algorithms were also compared in predicting mortality, 31 days mortality and 91 days mortality. The BI-LSTM algorithm model predicted the mortality and 91 days mortality with an accuracy of $80 \%$ and $94.2 \%$, respectively and outperformed other algorithms. The random forest algorithm model outperformed other algorithms with an accuracy of $98.9 \%$ in predicting 31 days of mortality. The BI-LSTM model predicted the 31 days mortality with an accuracy of $96.6 \%$.

Data analysis on mortality days shows that ASA grade, Complication, Curative Surgery, Additional procedures, Operation mode and Preoperative M stage are the variables that could be used to classify different groups of mortality days. $\mathrm{BMI}$ is not a good indicator in categorising different groups of mortality days. Figure 7 appears to 
show that a higher BMI leads to higher mortality days. However, this pattern is not correct as BMI groups 31-35 and $\leq 35$ only represent around $5 \%$ of the whole dataset. Moreover, data analysis of the dataset shows a 30 days mortality of $3.39 \%$ for CRC surgery, which is comparable with the results of other similar studies ranged between $0.9 \%$ and $9.9 \%[22,43,56]$.

\subsection{Limitations}

A limitation of this work has been the number of data samples, which were derived from a single centre. We are planning to collaborate with other colorectal groups to pool our data. Future work will therefore include a larger dataset comprising more samples and additional variables or features. Nevertheless, some clear patterns have emerged from the data in the current study.

\section{Conclusions}

Data analytics and AI have been shown to be accurate tools for predicting length of stay, readmission, and mortality following colorectal cancer surgery. Such predictive capability is important for designing the best patient care and prioritising resources.

These three proxies for patient outcomes were found to share the following common significant predictors: Age, Stoma, and Operation mode. LOS was also found to be a significant predictor for the other two patient outcomes, i.e., readmission and mortality. Other predictors had greater or lesser significance for each patient outcome. Nevertheless, the prediction algorithms were most effective when using the full data set rather than just the main predictors.

Bidirectional long short-term memory (BILSTM) was found to be the best prediction algorithm overall. In each case, we have demonstrated accuracies of greater than $80 \%$ and sensitivities and specificities of at least $84 \%$ and $75 \%$ respectively. The best results were achieved for 31 days mortality, with 96\% accuracy, 93\% sensitivity and $100 \%$ specificity. With improving techniques, richer data sets, and overlaid clinical expertise, further improvements can be anticipated, leading to improved patient outcomes and more efficient healthcare services.

\section{Declarations}

\section{Funding}

This work has been supported by the University of Portsmouth's Thematic Research \& Innovation Fund (TRIF).

\section{Conflicts of interest/Competing interests}

Not applicable.

\section{Data availability}

Enquiries about data access can be made via JK.

\section{Code availability}

The computer code has been deposited in the github repository [32]

\section{Author contributions}

$\mathrm{AH}$ and JK conceived the project idea. SM conducted the data analytics and machine learning under the supervision of AH. JK and his team (SS and KF) provided the data and clinical interpretation. SM wrote the original manuscript and provided all the figures. AH, SM, and JK reviewed and revised the article.

\section{References}

[1] Jamil Ahmed, Michael Lim, Shakeeb Khan, Claire McNaught, and John MacFie. Predictors of length of stay in patients having elective colorectal surgery within an enhanced recovery protocol. International Journal of Surgery, 8(8):628-632, 2010. https://doi.org/10.1016/j.ijsu.2010.07.294

[2] Arnaud Alves, Yves Panis, Pierre Mathieu, Fabrice Kwiatkowski, Karem Slim, and Georges Mantion. Mortality and morbidity after surgery of mid and low rectal cancer: results of a french prospective multicentric study. Gastroenterologie clinique et biologique, 29(5):509-514, $2005 . \quad$ https://doi.org/10.1016/s03998320(05)82121-9 
[3] Arnaud Alves, Yves Panis, Pierre Mathieu, Georges Mantion, Fabrice Kwiatkowski, and Karem Slim. Postoperative mortality and morbidity in french patients undergoing colorectal surgery: results of a prospective multicenter study. Archives of surgery, 140(3):278-283, 2005. https://doi.org/10.1001/archsurg.140.3.278

[4] Ariadni Aravani, Elizabeth F Samy, James D Thomas, Phil Quirke, Eva JA Morris, and Paul J Finan. A retrospective observational study of length of stay in hospital after colorectal cancer surgery in england (1998-2010). Medicine, 95(47), 2016. https://dx.doi.org/10.1097\%2FMD.0000000000005064

[5] National Bowel Cancer Audit. Annual Report 2019, 2020.

[6] Nahla Barakat, Andrew P Bradley, and Mohamed Nabil H Barakat. Intelligible support vector machines for diagnosis of diabetes mellitus. IEEE transactions on information technology in biomedicine, 14(4):1114-1120, 2010. https://doi.org/10.1109/titb.2009.2039485

[7] Michael Bauer, Les Fitzgerald, Emily Haesler, and Mara Manfrin. Hospital discharge planning for frail older people and their family. are we delivering best practice? a review of the evidence. Journal of clinical nursing, 18(18):25392546 , 2009. https://doi.org/10.1111/j.13652702.2008.02685.x

[8] James Bergstra and Yoshua Bengio. Random search for hyper-parameter optimization. The Journal of Machine Learning Research, 13(1):281-305, 2012. https://dl.acm.org/doi/10.5555/2188385.2188395

[9] Freddie Bray, Jacques Ferlay, Isabelle Soerjomataram, Rebecca L Siegel, Lindsey A Torre, and Ahmedin Jemal. Global cancer statistics 2018: Globocan estimates of incidence and mortality worldwide for 36 cancers in 185 countries. CA: a cancer journal for clinicians, 68(6):394-424, 2018. https://doi.org/10.3322/caac.21492
[10] Herng-Chia Chiu, Yi-Chieh Lin, Hui-Min Hsieh, Hsin-Pao Chen, Hui-Li Wang, and JawYuan Wang. The impact of complications on prolonged length of hospital stay after resection in colorectal cancer: A retrospective study of taiwanese patients. Journal of International Medical Research, 45(2):691-705, 2017. https://doi.org/10.1177/0300060516684087

[11] Jun Seong Chung, Han Deok Kwak, and Jae Kyun Ju. Thirty-day readmission after elective colorectal surgery for colon cancer: A single-center cohort study. Annals of coloproctology, 36(3):186, 2020. https://doi.org/10.3393/ac.2019.11.04

[12] GP Copeland, D Jones, and MPOSSUM Walters. Possum: a scoring system for surgical audit. British Journal of Surgery, 78(3):355-360, 1991. https://doi.org/10.1002/bjs.1800780327

[13] Rachelle N Damle and Karim Alavi. Risk factors for 30-d readmission after colorectal surgery: a systematic review. journal of surgical research, 200(1):200-207, 2016. https://doi.org/10.1016/j.jss.2015.06.052

[14] C Esteban, Inmaculada Arostegui, Javier Moraza, M Aburto, Jose M Quintana, Julio Pérez-Izquierdo, Susana Aizpiri, and Alberto Capelastegui. Development of a decision tree to assess the severity and prognosis of stable copd. European Respiratory Journal, 38(6):1294-1300, 2011. https://doi.org/10.1183/09031936.00189010

[15] Victor W Fazio, Paris P Tekkis, Feza Remzi, and Ian C Lavery. Assessment of operative risk in colorectal cancer surgery: the cleveland clinic foundation colorectal cancer model. Diseases of the colon \& rectum, 47(12):20152024, 2004. https://doi.org/10.1007/s10350004-0704-y

[16] Ali M Ferjani, Damian Griffin, Nigel Stallard, and Ling S Wong. A newly devised scoring system for prediction of mortality in patients with colorectal cancer: a prospective study. The lancet oncology, 8(4):317-322, $2007 . \quad$ https://doi.org/10.1016/s14702045(07)70045-1 
[17] Chloé Gervès-Pinquié, Anne Girault, Serena Phillips, Sarah Raskin, and Mandi Pratt-Chapman. Economic evaluation of patient navigation programs in colorectal cancer care, a systematic review. Health economics review, 8(1):12, 2018. https://dx.doi.org/10.1186\%2Fs13561-0180196-4

[18] Pierre Geurts, Damien Ernst, and Louis Wehenkel. Extremely randomized trees. Machine learning, 63(1):3-42, 2006. https:// doi.org/10.1007/s10994-006-6226-1

[19] Isabelle Guyon and André Elisseeff. An introduction to variable and feature selection. Journal of machine learning research, 3(Mar):1157-1182, 2003. https://doi.org/10.1162/153244303322753616

[20] Luke O Hansen, Robert S Young, Keiki Hinami, Alicia Leung, and Mark V Williams. Interventions to reduce 30-day rehospitalization: a systematic review. Annals of internal medicine, $155(8): 520-5 \overline{28,2011 .}$ https://doi.org/10.7326/0003-4819-155-8201110180-00008

[21] D Henneman, NJ Van Leersum, M Ten Berge, HS Snijders, M Fiocco, T Wiggers, RAEM Tollenaar, and MWJM Wouters. Failure-torescue after colorectal cancer surgery and the association with three structural hospital factors. Annals of surgical oncology, 20(11):33703376, 2013. https://doi.org/10.1245/s10434013-3037-z

[22] Daniel Henneman, Annelotte CM van Bommel, Alexander Snijders, Heleen S Snijders, Rob AEM Tollenaar, Michel WJM Wouters, and Marta Fiocco. Ranking and rankability of hospital postoperative mortality rates in colorectal cancer surgery. Annals of surgery, 259(5):844-849, 2014. https://doi.org/10.1097/sla.0000000000000561

[23] Mark C Hornbrook, Ran Goshen, Eran Choman, Maureen O'Keeffe-Rosetti, Yaron Kinar, Elizabeth G Liles, and Kristal C Rust. Early colorectal cancer detected by machine learning model using gender, age, and complete blood count data. Digestive diseases and sciences, 62(10):2719-2727, 2017. https://doi.org/10.1007/s10620-017-4722-8

[24] Stephen F Jencks. Defragmenting care. Annals of Internal Medicine, 153(11):757-758, 2010. https://doi.org/10.7326/0003-4819-15311-201012070-00010

[25] Maria Kelly, Linda Sharp, Fiona Dwane, Tracy Kelleher, and Harry Comber. Factors predicting hospital length-of-stay and readmission after colorectal resection: a populationbased study of elective and emergency admissions. BMC health services research, 12(1):77, 2012. https://doi.org/10.1186/1472-6963-1277

[26] Diederik P Kingma and Jimmy Ba. Adam: A method for stochastic optimization. arXiv preprint arXiv:1412.6980, 2014.

[27] Konstantina Kourou, Themis P Exarchos, Konstantinos P Exarchos, Michalis V Karamouzis, and Dimitrios I Fotiadis. Machine learning applications in cancer prognosis and prediction. Computational and structural biotechnology journal, 13:8-17, 2015. https://doi.org/10.1016/j.csbj.2014.11.005

[28] Max Kuhn and Kjell Johnson. Feature engineering and selection: A practical approach for predictive models. CRC Press, 2019. https://doi.org/10.1201/9781315108230

[29] Linda T Li, Whitney L Mills, Donna L White, Alexa Li, Amanda M Gutierrez, David H Berger, and Aanand D Naik. Causes and prevalence of unplanned readmissions after colorectal surgery: a systematic review and meta-analysis. Journal of the American Geriatrics Society, 61(7):1175-1181, 2013. https://doi.org/10.1111/jgs.12307

[30] Luiz Felipe de Campos Lobato, Patrícia Cristina Alves Ferreira, Elizabeth C Wick, Ravi P Kiran, Feza H Remzi, Matthew F Kalady, and Jon D Vogel. Risk factors for prolonged length of stay after colorectal surgery. Journal of Coloproctology (Rio de Janeiro), 33(1):22-27, 2013. 
https://dx.doi.org/10.1097\%2F00000658199908000-00016

[31] Angela B Mariotto, K Robin Yabroff, Yongwu Shao, Eric J Feuer, and Martin L Brown. Projections of the cost of cancer care in the united states: 2010-2020. Journal of the National Cancer Institute, 103(2):117-128, 2011. https://dx.doi.org/10.1093\%2Fjnci\%2Fdjq495

[32] S. Masum. Eventpredictions. https://github.com/shamsulmasum/ Colorectal_Feature_Analysis, April 2021.

[33] Shamsul Masum, John P Chiverton, Ying $\mathrm{Liu}$, and Branislav Vuksanovic. Investigation of machine learning techniques in forecasting of blood pressure time series data. In International Conference on Innovative Techniques and Applications of Artificial Intelligence, pages 269-282. Springer, 2019. https://doi.org/10.1007/978-3-030-34885-4_21

[34] Shamsul Masum, Ying Liu, and John Chiverton. Multi-step time series forecasting of electric load using machine learning models. In International Conference on Artificial Intelligence and Soft Computing, pages 148-159. Springer, 2018. https://doi.org/10.1007/978-3-319-91253-0_15

[35] Wes McKinney et al. Data structures for statistical computing in python. In Proceedings of the 9th Python in Science Conference, volume 445, pages 51-56. Austin, TX, 2010. https://doi.org/10.25080/Majora92bf1922-00a

[36] Andreas C Müller, Sarah Guido, et al. Introduction to machine learning with Python: a guide for data scientists. " O'Reilly Media, Inc.", 2016.

[37] AC Murray, C Mauro, J Rein, and RP Kiran. 30-day mortality after elective colorectal surgery can reasonably be predicted. Techniques in coloproctology, 20(8):567-576, 2016. https://doi.org/10.1007/s10151-016-1503-x
[38] National Cancer Intelligence Network. 30Day Post-Operative Mortality after Colorectal Cancer Surgery in England, 2011.

[39] Liyan Pan, Guangjian Liu, Fangqin Lin, Shuling Zhong, Huimin Xia, Xin Sun, and Huiying Liang. Machine learning applications for prediction of relapse in childhood acute lymphoblastic leukemia. Scientific reports, 7(1):19, 2017. https://doi.org/10.1038/s41598-01707408-0

[40] Ives Cavalcante Passos, Benson Mwangi, and Flávio Kapczinski. Big data analytics and machine learning: 2015 and beyond. The Lancet Psychiatry, 3(1):13-15, 2016 . https://doi.org/10.1016/s22150366(15)00549-0

[41] Fabian Pedregosa, Gaël Varoquaux, Alexandre Gramfort, Vincent Michel, Bertrand Thirion, Olivier Grisel, Mathieu Blondel, Peter Prettenhofer, Ron Weiss, Vincent Dubourg, et al. Scikit-learn: Machine learning in python. the Journal of machine Learning research, 12:2825-2830, 2011. https://doi.org/10.5555/1953048.2078195

[42] DR Prytherch, MS Whiteley, B Higgins, PC Weaver, WG Prout, and SJ Powell. Possum and portsmouth possum for predicting mortality. British Journal of Surgery, 85(9):121 $\overline{\text {-1220, }}$ 1998. https://doi.org/10.1046/j.13652168.1998.00840.x

[43] S Pucciarelli, M Zorzi, N Gennaro, G Gagliardi, Angelo Restivo, M Saugo, A Barina, M Rugge, M Zuin, I Maretto, et al. In-hospital mortality, 30-day readmission, and length of hospital stay after surgery for primary colorectal cancer: A national population-based study. European Journal of Surgical Oncology (EJSO), 43(7):1312-1323, 2017. https://doi.org/10.1016/j.ejso.2017.03.003

[44] Payam Refaeilzadeh, Lei Tang, and Huan Liu. Cross-validation. Encyclopedia of database systems, $5: 532-538,2009$. https://doi.org/10.1007/978-0-387-399409_565 
[45] Muni Rubens, Venkataraghavan Ramamoorthy, Anshul Saxena, Chintan Bhatt, Sankalp Das, Emir Veledar, Peter McGranaghan, Ana Viamonte-Ros, Yazmin Odia, Michael Chuong, et al. A risk model for prediction of 30day readmission rates after surgical treatment for colon cancer. International journal of colorectal disease, 35:1529-1535, 2020 . https://doi.org/10.1007/s00384-020-03605-y

[46] Eric B Schneider, Omar Hyder, Benjamin S Brooke, Jonathan Efron, John L Cameron, Barish H Edil, Richard D Schulick, Michael A Choti, Christopher L Wolfgang, and Timothy M Pawlik. Patient readmission and mortality after colorectal surgery for colon cancer: impact of length of stay relative to other clinical factors. Journal of the American College of Surgeons, 214(4):390-398, 2012. https://doi.org/10.1016/j.jamcollsurg.2011.12.025

[47] Anthony J Senagore, Anthony J Warmuth, Conor P Delaney, Paris $\mathrm{P}$ Tekkis, and Victor W Fazio. Possum, p-possum, and crpossum: implementation issues in a united states health care system for prediction of outcome for colon cancer resection. Diseases of the colon \& rectum, 47(9):1435- $\overline{1441,2004 .}$ https://doi.org/10.1007/s10350-004-0604-1

[48] Rebecca L Siegel, Kimberly D Miller, Ann Goding Sauer, Stacey A Fedewa, Lynn F Butterly, Joseph C Anderson, Andrea Cercek, Robert A Smith, and Ahmedin Jemal. Colorectal cancer statistics, 2020. CA: a cancer journal for clinicians, 2020. https://doi.org/10.3322/caac.21601

[49] Paris P Tekkis, Jan D Poloniecki, Michael R Thompson, and Jeffrey D Stamatakis. Operative mortality in colorectal cancer: prospective national study. Bmj, 327(7425):1196-1201, 2003. https://doi.org/10.1136/bmj.327.7425.1196

[50] PP Tekkis, DR Prytherch, HM Kocher, A Senapati, JD Poloniecki, JD Stamatakis, and ACJ Windsor. Development of a dedicated risk-adjustment scoring system for colorectal surgery (colorectal possum). British Journal of Surgery, 91(9):1174-1182, 2004. https://doi.org/10.1002/bjs.4430

[51] Sarah E Tevis, Evie H Carchman, Eugene F Foley, Bruce A Harms, Charles P Heise, and Gregory D Kennedy. Postoperative ileus-more than just prolonged length of stay? Journal of Gastrointestinal Surgery, $\quad 19(9): 1684-1690, \quad 2015$. https://doi.org/10.1007/s11605-015-2877-1

[52] J William Thomas and James J Holloway. Investigating early readmission as an indicator for quality of care studies. Medical care, pages 377-394, 1991. https://doi.org/10.1097/00005650-19910400000006

[53] Robert Tibshirani. Regression shrinkage and selection via the lasso. Journal of the Royal Statistical Society: Series B (Methodological), 58(1):267-288, 1996. https://doi.org/10.1111/j.25176161.1996.tb02080.x

[54] Cancer Research UK. Bowel cancer statistics, 2018.

[55] FJ Van Der Sluis, E Espin, F Vallribera, GH de Bock, HJ Hoekstra, BL Van Leeuwen, and AF Engel. Predicting postoperative mortality after colorectal surgery: a novel clinical model. Colorectal Disease, 16(8):631-639, 2014. https://doi.org/10.1111/codi.12580

[56] Elmer E van Eeghen, Frank C den Boer, and Ruud JLF Loffeld. Thirty days postoperative mortality after surgery for colorectal cancer: a descriptive study. Journal of gastrointestinal oncology, 6(6): $\overline{613,2015 .}$ https://dx.doi.org/10.3978\%2Fj.issn.20786891.2015.079

[57] Don Vicendese, Luc Te Marvelde, Peter D McNair, Kathryn Whitfield, Dallas R English, Souhaib Ben Taieb, Rob J Hyndman, and Robert Thomas. Hospital characteristics, rather than surgical volume, predict length of stay following colorectal cancer surgery. Australian and New Zealand journal of public health, 44(1):73-82, 2020. https://doi.org/10.1111/1753-6405.12932 
[58] S Wilkins, K Oliva, E Chowdhury, B Ruggiero, A Bennett, EJ Andrews, O Dent, $\mathrm{P}$ Chapuis, C Platell, CM Reid, et al. Australasian acpgbi risk prediction model for 30-day mortality after colorectal cancer surgery. BJS open, 4(6):1208, 2020. https://doi.org/ $\overline{10.1002 / \text { bjs } 5.50356}$

[59] DJ Williams and JD Walker. A nomogram to calculate the physiological and operative severity score for the enumeration of mortality and morbidity (possum). British Journal of Surgery, 101(3):239-245, 2014. https://doi.org/10.1002/bjs.9363

[60] Yucan Xu, Lingsha Ju, Jianhua Tong, ChengMao Zhou, and Jian-Jun Yang. Machine learning algorithms for predicting the recurrence of stage iv colorectal cancer after tumor resection. Scientific reports, 10(1):1-9, 2020. https://doi.org/10.1038/s41598-020-59115-y

[61] In-Kwon Yeo and Richard A Johnson. A new family of power transformations to improve normality or symmetry. Biometrika, 87(4):954-959, 2000. https://doi.org/10.1093/biomet/87.4.954 